\title{
Schluss: Erfolgreiche Zusammenarbeit
}

Die Unterschiede zwischen dem Arbeits- und Managementstil in China und Deutschland sind für beide Seiten offensichtlich. Die Durchführung systematischer Studien oder die Auswertung bestehender Daten zu den Bereichen Personal und Führung sind in China für inländische wie ausländische Partner aktuell nur mit wenigen Ausnahmen möglich. In unserer Darstellung zur Entwicklung der Führungskräfte war es unser Anliegen, wesentliche Charakteristika der chinesischen Herangehensweise herauszuarbeiten. So stellen wir dem Leser erste Eindrücke über die konkreten Unterschiede im Arbeitsalltag vor. Dabei greifen wir auf unsere jahrelange Felderfahrung bei der Begleitung von Führungskräften beider Länder zurück.

Eine häufig gestellte Frage unserer Trainingsteilnehmer ist, ob es nötig ist, sich an die chinesischen Vorstellungen anzupassen. Unsere Antwort ist mehr eine Empfehlung: Wenn es Ihnen gelingt, sich in wichtigen Punkten in Ihrem Verhalten an die chinesischen Wertvorstellungen anzunähern, erleichtert Ihnen das die Zusammenarbeit nachhaltig.

Warum? Ihren chinesischen Partnern wird es meist an internationalen Erfahrungen fehlen. Sie sind sicher noch nicht geübt darin, Ihr Verhalten unter dem Aspekt der deutschen Kultur zu beurteilen. Bieten Sie ein unter chinesischen Aspekten geschätztes Bild Ihrer Persönlichkeit, kann dies Missverständnisse und anhaltende Störungen in der Zusammenarbeit vermeiden. Dabei soll es natürlich nicht darum gehen, unreflektiert chinesisches Verhalten zu kopieren. Aus unserer Sicht steht es eher im Vordergrund, Ihren Blick für die Wertvorstellungen Ihrer Partner zu schärfen und die vorgestellten Informationen zu Ihrem Vorteil zu nutzen.

Wir wünschen Ihnen bei der Entfaltung Ihres Potenzials alles Gute! 